\title{
COBORDISM OF GROUP ACTIONS
}

\author{
BY ARTHUR WASSERMAN ${ }^{1}$
}

Communicated by R. Palais, May 9, 1966

Let $G$ be a compact Lie group and $M$ a compact $G$ manifold without boundary, i.e. a $C^{\infty}$ manifold with a differentiable action of $G$ on $M . M^{n}$ is said to be $G$-cobordant to zero $M \sim_{G} 0$ if there exists a compact $G$ manifold $Q^{n+1}$ with $\partial Q=M$. Note that in this case $M_{G}$ (the fixed point set of $M)=\partial Q_{G} . M_{G}$ and $Q_{G}$ are both disjoint unions of closed submanifolds (of varying dimension) of $M, Q$ respectively. Let $\nu\left(M_{G}, M\right)$ denote the normal bundle of $M_{G}$ in $M ; \nu\left(M_{G}, M\right) \rightarrow M_{G}$ is a $G$-vector bundle in the sense of [5]. A partial converse to the statement $\nu\left(M_{G}, M\right)=\partial \nu\left(Q_{G}, Q\right)$ is given by

Proposition 1 ([2, p. 10]). If $\nu\left(M_{G}, M\right)$ is cobordant to zero as a $G$-vector bundle, i.e. if there exists a manifold $W$ and a $G$-vector bundle $E \rightarrow W$ with $\partial W=M_{G}, E \mid \partial W=\nu\left(M_{G}, M\right)$ then $M$ is G-cobordant to a manifold $M^{\prime}$ with $M_{G}^{\prime}=\varnothing$.

Proof. Form the manifold $M \times I \cup_{f} E(1)$ where $E(1)$ denotes the unit disc bundle in $E$ and

Then note that, after smoothing,

$$
f: E(1) \mid \partial W=\nu\left(M_{G}, M\right) \underset{\mathrm{fix}}{\stackrel{\exp }{\longrightarrow}} M \times 1 .
$$

$$
\begin{aligned}
\partial\left(M \times I \bigcup_{f} E(1)\right) & =M \times 0 \cup(M \times 1-f(E(1) \mid \partial W) \cup \partial E(1)) \\
& =M \times 0 \cup M^{\prime} .
\end{aligned}
$$

Hence, one may view the $G$-cobordism class of $\nu\left(M_{G}, M\right)$ as a first obstruction to finding a cobordism $M \sim_{G} 0$. Higher obstructions are formulated in terms of a spectral sequence. For simplicity we deal only with the unoriented case.

Let $V$ be an orthogonal representation of $G$ and let $V^{n}$ denote the $n$-fold direct sum of $V$ with itself and $S(V)$ the unit sphere in $V$. Consider the category of manifolds $\mathcal{G}(V)$ where $M$ is in $\mathcal{G}(V)$ iff $M$ can be imbedded in $S\left(V^{n}\right)$ for some $n$. One can then define the cobordism groups $\mathfrak{T}_{n}(V)=\mathfrak{T}_{n}(\mathcal{G}(V))$ of $n$ dimensional $G$-manifolds in $g(V)$ (see [5]). It was shown in [5] that if $G$ is finite or abelian then $\mathfrak{Y}_{n}(V) \approx \pi_{1}^{V^{2 n+3}}\left(T_{k}\left(V^{2 n+3} \oplus R\right), \infty\right)$ where $\pi_{1}^{\Gamma^{2 n+3}}\left(T_{k}\left(V^{2 n+3} \oplus R\right), \infty\right)$ de-

1 This research was supported in part by the U. S. Army Office of Research, (Durham). 
notes the equivariant homotopy classes of maps of $S\left(V^{2 n+3} \oplus R\right)$ into $T_{k}\left(V^{2 n+3} \oplus R\right)$ the Thom space of the universal bundle of $k$-planes in $V^{2 n+3} \oplus R$. Let $f$ be such a map; then proposition 1 may be reinterpreted as saying

Proposition 1'. Any homotopy of

$$
f \mid S\left(V^{2 n+3} \oplus R\right)_{G}: S\left(V^{2 n+3} \oplus R\right)_{G} \rightarrow T_{k}\left(V^{2 n+3} \oplus R\right)_{G}
$$

may be covered by a homotopy of $f$.

It was shown in [5] that there are only a finite number of conjugacy classes of isotropy groups occurring in $g(V)$; let $\left(H_{1}\right), \cdots$, $\left(H_{r}\right)$ denote the conjugacy classes ordered by $\left(H_{i}\right)<\left(H_{j}\right)$ iff there is a $g \in G$ with $g H_{i} g^{-1} \subset H_{j}$ but $g H_{i} g^{-1} \neq H_{j}$. Define the level $H_{i}>n$ if $H_{i}<H_{j}$ and level $H_{j}>n-1$; level $G=0$ by definition and level $H_{i}=n$ if level $H_{i}>n-1$ but not level $H_{i}>n$. We may filter $g(V)$ by subcategories $\mathcal{G}^{i}(V)$ where $M$ is in $\mathcal{G}^{i}(V)$ if for each $x \in M$ level $\left(G_{x}\right) \geqq i$ and $G_{x}$ is the isotropy group of $x$. One then has the corresponding cobordism groups $D_{n, i}=\mathscr{N}_{n}\left(\mathcal{G}^{i}(V)\right)$. Let $D_{n, i}=D_{n, 0}$ for $i \leqq 0$ and let $D^{n, i}$ denote the image of $D_{n, i}$ in $D_{n, 0}=\mathfrak{N}_{n}(V)$. We define $E_{n, i} n \geqq 0, i \geqq 0$, as the cobordism group of differentiable $G$-vector bundles $E \rightarrow M$ where $M$ is a compact $G$-manifold and

(i) $\operatorname{dim} E=n$;

(ii) $E$ is in $g(V)$;

(iii) $S(E)$ is in $\mathcal{G}(V)^{i+1}$ where $S(E)$ is the unit sphere bundle in $E$;

(iv) level $\left(G_{x}\right)=i$ for all $x \in M$.

Define $E_{n, i}=0$ for $i<0$. Vector bundles with fibre dimension zero are included.

THEOREM. There is a graded exact couple

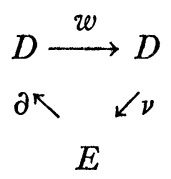

where

$$
D=\sum_{n, i} D_{n, i}, \quad E=\sum_{n, i} E_{n, i}
$$

with

$$
E_{n, i}^{r} \Rightarrow E_{n, i}^{\infty}=D^{n, i} / D^{n, i+1}
$$


In particular

$$
\mathfrak{N}_{n}(V) \approx \sum_{i=0}^{\infty} E_{n, i}^{\infty} .
$$

The maps are as follows: Define $w: D_{n, i} \rightarrow D_{n, i-1}$ by $w([M])=[M]$; if $M$ is in $g^{i}(V)$ then $M$ is in $g^{i-1}(V)$. Define $\partial: E_{n, i} \rightarrow D_{n-1, i+1}$ by $\partial(E \rightarrow M)=S(E) ; \partial$ is well defined by (i), (ii), and (iii). Define $\nu: D_{n, i} \rightarrow E_{n, i}$ by $\nu([M])=\left[\nu\left(M_{i}, M\right)\right]$ where $M_{i}=\left\{x \in M \mid\right.$ level $\left(G_{x}\right)$ $=i\} ; M_{i}$ is a closed submanifold since $M$ is in $\mathcal{G}^{i}(V)$. Conditions (i)-(iv) are clearly satisfied. Exactness follows from straightforward geometric arguments.

The groups $E_{n, i}$ may be described as follows: let $H$ be an isotropy group on level $i$ and let $W$ be an $r$ dimensional representation of $H$ with $W \subset V^{s} \mid H$ for some $s$ where $V^{s} \mid H$ means $V^{s}$ considered as an $H$ space.

Let $P(H, W)$ be the group of $N(H)$ (normalizer of $H$ in $G$ ) equivariant bundle maps of $W \times_{H} N(H)$ in to itself which are diffeomorphisms on the base space $N(H) / H$. We have the exact sequence $0 \rightarrow O_{H}(W) \rightarrow P(H, W) \rightarrow N(H) / H \rightarrow 0$ where $O_{H}(W)$ is the group of $H$ equivariant orthogonal transformations of $W$.

Proposition 2. $E_{n, i}$ is isomorphic to the direct sum of $\Re_{t}(B P(H, W))$ over all such representations of $H$ and all conjugacy classes of subgroups on level $i ; \Re_{t}(B P(H, W)$ ) denotes the ordinary cobordism group (see $[1$, p. 45]) of the classifying space of $P(H, W)$ and $t=n-\operatorname{dim} W$ $-\operatorname{dim} G / H$.

Proof. Let $E \rightarrow M$ be a bundle in $E_{n, i}$ with $\left(G_{x}\right)=(H)$ for all $x \in M$. By equivariance, it suffices to consider the $N(H)$ bundle $E \mid M_{H}$ $\rightarrow M_{H}\left(M_{H}=\left\{x \in M \mid G_{x}=H\right\}\right)$ since $M=M_{H} \times_{N(H)} G([3, \mathrm{p} .42])$; but $M_{H}$ is a $N(H) / H$ principal bundle over $M / G$ and hence one can see that $E \mid M_{H} \rightarrow M_{H} \rightarrow M / G$ is an $N(H)$ fibre bundle with fibre $N(H) \times_{H} W$ and structural group $P(H, W)$ ([4, p. 40]). Any element of $E_{n, i}$ is the disjoint union of such bundles.

To describe the differential we let $K \subset H$ be an isotropy group on level $i+1$; then $W \mid K=W_{0} \oplus W_{1}$ where $K$ operates trivially on $W_{0}$. $S\left(W_{0}\right)$ is a $N(K, H) / K$ principal bundle where $N(K, H)$ denotes the normalizer of $K$ in $H$. Form the $N(H)$ bundle $U$ over $B P(H, W)$ with fibre $N(H) \times_{H} S(W), \quad U=E_{P} \times_{P}\left(N(H) \times_{H} S(W)\right)$ where $E_{P}$ $\rightarrow B P(H, W)$ is the universal principal bundle; then $U_{(K)} / N(H)$ $=U(K)$ is a bundle over $B P(H, W)$ with fibre $S\left(W_{0}\right) / N(K, H)$ and there is a map $i: U(K) \rightarrow B P\left(K, W_{1}\right)$ which classifies the normal 
bundle of $U_{(K)}$ in $U$. Then for any $[M, f] \in \mathfrak{K}_{t}(B P(H, W))$ we have the diagram

$$
\begin{gathered}
f^{*} U(K) \stackrel{f_{*}}{\rightarrow} U(K) \stackrel{i}{\rightarrow} B P\left(K, W_{1}\right) \\
\downarrow \\
\quad \stackrel{f}{\rightarrow} B P(H, W) .
\end{gathered}
$$

Clearly $d([M, f])=\sum\left[f^{*} U(K), i \circ f_{*}\right] \in E_{n-1, i+1}$ where $\left[f^{*} U(K)\right.$, $\left.i \circ f_{*}\right] \in \Re_{s}\left(B P\left(K, W_{1}\right), s=n-1-\operatorname{dim} W_{1}-\mathrm{im} G / K\right.$, and the sum extends over all conjugacy classes $(K)$ on level $i+1$ with $K \subset H$.

\section{REFERENCES}

1. P. E. Conner and E. E. Floyd, Differentiable periodic maps, Academic Press, New York, 1964.

2. - - Supplement to differentiable periodic maps, Academic Press, New York, 1964.

3. R. Palais, The classification of G-spaces, Mem. Amer. Math. Soc., No. 36 (1960).

4. N. Steenrod, The topology of fiber bundles, Princeton Univ. Press, Princeton, N. J., 1951.

5. A. Wasserman, Equivariant differential topology (to appear).

HARVARD UNIVERSITY 